\title{
Boundary-Domain Integral Equations for Variable Coefficient Dirichlet BVP in 2D Unbounded Domain
}

\author{
T. T. Dufera and S. E. Mikhailov
}

\begin{abstract}
In this paper, the Dirichlet boundary value problem for the second order stationary diffusion elliptic partial differential equation with variable coefficient is considered in unbounded (exterior) two dimensional domain. Using an appropriate parametrix (Levi function), this problem is reduced to some direct segregated Boundary-Domain Integral Equations (BDIEs). We investigate the properties of corresponding parametrix-based integral volume and layer potentials in some weighted Sobolev spaces, as well as the unique sovability of BDIEs and their equivalence to the original BVP.
\end{abstract}

\section{Basic Notations and Function Spaces}

Let $\Omega=\Omega^{+}$be an unbounded open domain in $\mathbb{R}^{2}$ such that the complement $\Omega^{-}:=\mathbb{R}^{2} \backslash \bar{\Omega}$ is bounded open domain. Let the boundary $\partial \Omega=\partial \Omega^{-}$be closed and infinitely smooth curve. The space of infinitely differentiable functions having compact support in $\Omega$ is denotes by $\mathscr{D}(\Omega)$ and its dual space, the space of distributions, by $\mathscr{D}^{\prime}(\Omega)$, while $\mathscr{D}(\bar{\Omega})$ is the set of restrictions on $\bar{\Omega}$ of functions from $\mathscr{D}\left(\mathbb{R}^{2}\right)$. The spaces $H^{s}(\Omega), H^{s}(\partial \Omega)$ denote the Sobolev (Bessel potential) spaces.

We shall consider the following second order partial differential equation, with variable coefficient

$$
A u(x):=\sum_{i=1}^{2} \frac{\partial}{\partial x_{i}}\left(a(x) \frac{\partial u(x)}{\partial x_{i}}\right)=f(x) \quad x \in \Omega
$$

T.T. Dufera

Adama Science and Technology University, Adama, Ethiopia

e-mail: tamirat.temesgen@astu.edu.et

S.E. Mikhailov

Brunel University London, UK

e-mail: sergey.mikhailov@brunel.ac.uk 
where $u$ is unknown function; $f(x)$ and $a(x)>a_{0}>0$ are given functions in $\Omega$.

We will further use the weighted Sobolev spaces. Let

$$
\rho_{2}(x):=\left(1+|x|^{2}\right)^{1 / 2} \ln \left(2+|x|^{2}\right) .
$$

For any real $\beta$, we denote by $L_{2}\left(\rho_{2}^{\beta} ; \Omega\right)$ the weighted Lebesgue space (see, e.g., [7]) consisting of all measurable functions $g(x)$ on $\Omega$ such that $g \rho_{2}^{\beta} \in L_{2}(\Omega)$, i.e.,

$$
\|g\|_{L_{2}\left(\rho_{2}^{\beta} ; \Omega\right)}^{2}=\int_{\Omega}\left|g(x) \rho_{2}^{\beta}(x)\right|^{2} d x<\infty .
$$

The space $L_{2}\left(\rho_{2}^{\beta} ; \Omega\right)$, equipped with the norm $\|\cdot\|_{L_{2}\left(\rho_{2}^{\beta} ; \Omega\right)}$ and appropriate inner product, is a Hilbert space.

The weighted Sobolev space $\mathscr{H}^{1}(\Omega)$ is defined by

$$
\mathscr{H}^{1}(\Omega):=\left\{g \in L_{2}\left(\rho_{2}^{-1} ; \Omega\right): \nabla g \in L_{2}(\Omega)\right\},
$$

and for its norm we have $\|g\|_{\mathscr{H}^{1}(\Omega)}^{2}:=\|g\|_{L_{2}\left(\rho_{2}^{-1} ; \Omega\right)}^{2}+\|\nabla g\|_{L_{2}(\Omega)}^{2}$, while $|g|_{\mathscr{H}^{1}(\Omega)}^{2}:=$ $\sum_{i=1}^{2} \int_{\Omega}\left|\frac{\partial g}{\partial x_{i}}\right|^{2} d x=\|\nabla g\|_{L_{2}(\Omega)}^{2}$ is the square of the semi-norm. The space $\mathscr{D}\left(\mathbb{R}^{2}\right)$ is dense in $\mathscr{H}^{1}\left(\mathbb{R}^{2}\right)$, see e.g., [1, Theorem 7.2]. This implies that the dual space of $\mathscr{H}^{1}\left(\mathbb{R}^{2}\right)$, denoted by $\mathscr{H}^{-1}\left(\mathbb{R}^{2}\right)$, is a space of distributions. Using the corresponding property for the space $H^{1}(\Omega)$, one can prove that $\mathscr{D}(\bar{\Omega})$ is dense in $\mathscr{H}^{1}(\Omega)$. The trace operator $\gamma^{+}$on $\partial \Omega$ defined on functions from $\mathscr{H}^{1}(\Omega)$, satisfies the usual trace theorems. This allows to define in particular the subspace

$$
\mathscr{H}_{0}^{1}(\Omega)=\left\{g \in \mathscr{H}^{1}(\Omega): \gamma^{+} g=0\right\} .
$$

It can be proved that $\mathscr{D}(\Omega)$ is dense in $\mathscr{H}_{0}^{1}(\Omega)$ and therefore, its dual space is a space of distributions. Let us denote by $\widetilde{\mathscr{H}}^{1}(\Omega)$ a completion of $\mathscr{D}(\Omega)$ in $\mathscr{H}^{1}\left(\mathbb{R}^{2}\right)$, and $\widetilde{\mathscr{H}}^{-1}(\Omega):=\left[\mathscr{H}^{1}(\Omega)\right]^{\prime}, \quad \mathscr{H}^{-1}(\Omega):=\left[\widetilde{\mathscr{H}}^{1}(\Omega)\right]^{\prime}$ are the corresponding dual spaces. The inclusion $L_{2}\left(\rho_{2} ; \Omega\right) \subset \mathscr{H}^{-1}(\Omega)$ holds and a distribution $f$ in the dual space $\widetilde{\mathscr{H}}^{-1}(\Omega)$ has the form $f=\sum_{i=1}^{2} \frac{\partial g_{i}}{\partial x_{i}}+f_{0}$, where $g_{i} \in L_{2}\left(\mathbb{R}^{2}\right)$ and is zero outside $\Omega, f_{0} \in L_{2}\left(\rho_{2} ; \Omega\right)$, cf. e.g., [12, Eq. (2.5.129)]. This implies that $\mathscr{D}(\Omega)$ is dense in $\mathscr{\mathscr { H }}^{-1}(\Omega)$ and $\mathscr{D}\left(\mathbb{R}^{2}\right)$ is dense in $\mathscr{H}^{-1}\left(\mathbb{R}^{2}\right)$.

From Definition (3) we obtain the following assertion.

Lemma 1. The space $\mathscr{H}^{1}(\Omega)$ contains constant functions.

Lemma 1 implies that, the space of real constants, $\mathbb{R}$, is a closed subspace of $\mathscr{H}^{1}(\Omega)$. Thus we can define the quotient space $\mathscr{H}^{1}(\Omega) / \mathbb{R}$, which is a Banach space, and its norm is given by $\|u+\mathbb{R}\|_{\mathscr{H}^{1}(\Omega) / \mathbb{R}}=\inf _{c \in \mathbb{R}}\|u+c\|_{\mathscr{H}^{1}(\Omega)}$. The dual space $\left(\mathscr{H}^{1}(\Omega) / \mathbb{R}\right)^{\prime}$ is identified with $\widetilde{\mathscr{H}}^{-1}(\Omega) \perp \mathbb{R}$, i.e., $\left(\mathscr{H}^{1}(\Omega) / \mathbb{R}\right)^{\prime}=$ $\widetilde{\mathscr{H}}^{-1}(\Omega) \perp \mathbb{R}$ since they are isometrically isomorphic (see e.g., [8, Lemma 2.12(ii)]). Similarly, $\left(\widetilde{\mathscr{H}^{1}}(\Omega) / \mathbb{R}\right)^{\prime}=\mathscr{H}^{-1}(\Omega) \perp \mathbb{R}$. 
The following Poincaré-type inequalities hold (cf. [2, Theorems 1.1 and 1.2]).

Theorem 1. (i) The semi-norm $|\cdot|_{\mathscr{H}^{1}(\Omega)}$ defined on $\mathscr{H}^{1}(\Omega) / \mathbb{R}$ is a norm equivalent to the quotient norm, i.e., there exist positive constants $c_{1}, C_{1}$ such that

$$
c_{1}|v|_{\mathscr{H}^{1}(\Omega)} \leq\|v\|_{\mathscr{H}^{1}(\Omega) / \mathbb{R}} \leq C_{1}|v|_{\mathscr{H}^{1}(\Omega)} .
$$

(ii) Moreover, the semi-norm $|\cdot|_{\mathscr{H}}^{1}(\Omega)$ is a norm on $\mathscr{H}_{0}^{1}(\Omega)$ equivalent to the norm $\|\cdot\|_{\mathscr{H}^{1}(\Omega)}$, i.e., there exist positive constants $c_{2}, C_{2}$ such that

$$
c_{2}|v|_{\mathscr{H}^{1}(\Omega)} \leq\|v\|_{\mathscr{H}_{0}^{1}(\Omega)} \leq C_{2}|v|_{\mathscr{H}^{1}(\Omega)}
$$

For $u \in \mathscr{H}^{1}(\Omega)$ and the coefficient $a(x) \in L_{\infty}(\Omega), \operatorname{PDE}(1)$ is well defined in the distributional sense as $\langle A u, v\rangle_{\Omega}:=-\langle a \nabla u, \nabla v\rangle_{\Omega}=-\mathscr{E}(u, v)$, for any $v \in \mathscr{D}(\Omega)$, where $\mathscr{E}(u, v):=\int_{\Omega} E(u, v)(x) d x, \quad E(u, v)(x):=\nabla v(x) \cdot a(x) \nabla u(x)$. Unless stated otherwise we henceforth assume that there are some constants $a_{0}, a_{1}$ such that

$$
a \in L_{\infty}\left(\mathbb{R}^{2}\right) \text { and } 0<a_{0}<a(x)<a_{1}<\infty \text { for a.e } x \in \mathbb{R}^{2} .
$$

To obtain boundary-domain integral equations, we will also always consider the coefficient $a$ such that

$$
a \in C^{1}\left(\mathbb{R}^{2}\right) \text { and } \rho_{2} \nabla a \in L_{\infty}\left(\mathbb{R}^{2}\right) .
$$

If $u \in H^{1}(\Omega)$, then $u \in \mathscr{H}^{1}(\Omega)$, from the trace theorem it follows that, $\gamma^{+} u \in$ $H^{\frac{1}{2}}(\partial \Omega)$, where $\gamma^{+}=\gamma_{\partial \Omega}^{+}$is the trace operator on $\partial \Omega$ from the exterior domain $\Omega^{+}$.

For the operator $A$, similar to [4] for the three dimensional case, we introduce the space, $\mathscr{H}^{1,0}(\Omega ; A):=\left\{g \in \mathscr{H}^{1}(\Omega): A g \in L_{2}\left(\rho_{2} ; \Omega\right)\right\}$, where the norm is given by its square, $\|g\|_{\mathscr{H}^{1,0}(\Omega ; A)}^{2}:=\|g\|_{\mathscr{C}^{1}(\Omega)}^{2}+\|A g\|_{L_{2}\left(\rho_{2} ; \Omega\right)}^{2}$. For $u \in \mathscr{H}^{1,0}(\Omega ; A)$, as in the 3D case, [4], we define the canonical co-normal derivative $T^{+} u \in H^{-\frac{1}{2}}(\partial \Omega)$ similar to, for example in [5, Lemma 3.2] and [8, Lemma 4.3] as

$$
\left\langle T^{+} u, \omega\right\rangle_{\partial \Omega}:=\int_{\Omega}\left[\left(\gamma_{-1}^{+} \omega\right) A u+E\left(u, \gamma_{-1}^{+} \omega\right)\right] d x \quad \forall \omega \in H^{\frac{1}{2}}(\partial \Omega),
$$

where $\gamma_{-1}^{+}: H^{\frac{1}{2}}(\partial \Omega) \rightarrow \mathscr{H}^{1}(\Omega)$ is a bounded right inverse to the trace operator $\gamma^{+}: \mathscr{H}^{1}(\Omega) \rightarrow H^{\frac{1}{2}}(\partial \Omega)$, and $\langle\cdot, \cdot\rangle_{\partial \Omega}$ denotes the duality brackets between the spaces $H^{-\frac{1}{2}}(\partial \Omega)$ and $H^{\frac{1}{2}}(\partial \Omega)$ which extends the usual $L_{2}(\partial \Omega)$ scalar product. The operator $T^{+}: \mathscr{H}^{1,0}(\Omega ; A) \rightarrow H^{-\frac{1}{2}}(\partial \Omega)$ is continuous and gives the continuous extension to $\mathscr{H}^{1,0}(\Omega ; A)$ of the classical co-normal derivative operator $a \frac{\partial}{\partial n}$, where $\frac{\partial}{\partial n}=\gamma^{+} \nabla \cdot n$ and $n=n^{+}$is normal vector on $\partial \Omega$ directed outward the exterior domain $\Omega$.

Similar to the proofs available in [5, Lemma 3.4] (see also [10] for the spaces $\left.H^{s, t}(\Omega ; A)\right)$, one can prove that for $u \in \mathscr{H}^{1,0}(\Omega ; A)$ the first Green identity 


$$
\left\langle T^{+} u, \gamma^{+} v\right\rangle_{\partial \Omega}=\int_{\Omega}[v A u+E(u, v)] d x \quad \forall v \in \mathscr{H}^{1}(\Omega)
$$

holds true. Then, for any functions $u, v \in \mathscr{H}^{1,0}(\Omega ; A)$ we have the second Green identity,

$$
\int_{\Omega}[v A u-u A v] d x=\left\langle T^{+} u, \gamma^{+} v\right\rangle_{\partial \Omega}-\left\langle T^{+} v, \gamma^{+} u\right\rangle_{\partial \Omega}
$$

Remark 1. If $a$ satisfies condition (4) and the second condition in (5), then $\|g a\|_{\mathscr{H}^{1}(\Omega)} \leq$ $C_{1}\|g\|_{\mathscr{H}^{1}(\Omega)},\left\|g \frac{1}{a}\right\|_{\mathscr{H}^{1}(\Omega)} \leq C_{2}\|g\|_{\mathscr{H}^{1}(\Omega)}$, where the constant $C_{1}$ and $C_{2}$ are independent of $g \in \mathscr{H}^{1}(\Omega)$, this means, $a$ and $1 / a$ are multipliers in the space $\mathscr{H}^{1}(\Omega)$.

Let us introduce the following subspaces

$$
\begin{aligned}
& L_{2}\left(\rho_{2} ; \Omega\right) \perp \mathbb{R}:=\left\{f \in L_{2}\left(\rho_{2} ; \Omega\right):\langle f, 1\rangle_{\Omega}=0\right\} \\
& \mathscr{H}^{1,0 \perp}(\Omega ; A):=\left\{g \in \mathscr{H}^{1}(\Omega): A g \in L_{2}\left(\rho_{2} ; \Omega\right) \perp \mathbb{R}\right\}, \\
& H_{*}^{-\frac{1}{2}}(\partial \Omega):=\left\{\psi \in H^{-\frac{1}{2}}(\partial \Omega):\langle\psi, 1\rangle_{\partial \Omega}=0\right\} .
\end{aligned}
$$

Employing the first Green identity (7) with $v=1$, we arrive at the following assertion.

Lemma 2. If $u \in \mathscr{H}^{1,0 \perp}(\Omega ; A)$ then $T^{+} u \in H_{*}^{-\frac{1}{2}}(\partial \Omega)$.

\section{Dirichlet BVP in Exterior Domain}

Given $f \in L_{2}\left(\rho_{2} ; \Omega\right)$ and $\varphi_{0} \in H^{\frac{1}{2}}(\partial \Omega)$, find a function $u \in \mathscr{H}^{1,0}(\Omega ; A)$ such that:

$$
\begin{gathered}
A u=f \quad \text { in } \Omega, \\
\gamma^{+} u=\varphi_{0} \quad \text { on } \partial \Omega .
\end{gathered}
$$

Let us denote by $\mathscr{A}_{D}=\left[A, \gamma^{+}\right]^{T}: \mathscr{H}^{1,0}(\Omega ; A) \rightarrow L_{2}\left(\rho_{2} ; \Omega\right) \times H^{\frac{1}{2}}(\partial \Omega)$, the lefthand side operator, which is evidently continuous. Similar to the proof in [4] for the three-dimensional case, one can prove the following assertion in the $2 \mathrm{D}$ case.

Theorem 2. Under conditions (4), the Dirichlet problem (9)-(10) is uniquely solvable and its solution can be written as $u=\mathscr{A}_{D}^{-1}\left(f, \varphi_{0}\right)^{T}$, where the operator $\mathscr{A}_{D}^{-1}: L_{2}\left(\rho_{2} ; \Omega\right) \times H^{\frac{1}{2}}(\partial \Omega) \rightarrow \mathscr{H}^{1,0}(\Omega)$ is continuous.

\section{Parametrix-Based Potentials in Exterior Domain}

A function $P(x, y)$ is a parametrix (Levi function) for the operator $A$ if $A_{x} P(x, y)=$ $\delta(x-y)+R(x, y)$, where $\delta$ is the Dirac-delta distribution, while $R(x, y)$ is a remainder possessing at most a weak (integrable) singularity at $x=y$. In particular, see e.g., 
[9] the function

$$
P(x, y)=\frac{\ln |x-y|}{2 \pi a(y)}, \quad x, y \in \mathbb{R}^{2},
$$

is a parametrix for the operator $A$ and the corresponding remainder is given by

$$
R(x, y)=\sum_{i=1}^{2} \frac{x_{i}-y_{i}}{2 \pi a(y)|x-y|^{2}} \frac{\partial a(x)}{\partial x_{i}}, \quad x, y \in \mathbb{R}^{2} .
$$

Let $u \in \mathscr{D}(\bar{\Omega})$. For any fixed $y \in \Omega$, let $B_{\varepsilon}(y)$ be an open ball centered at $y$ with a sufficiently small radius $\varepsilon>0$, and let $B_{r}(0)$ be an open ball centered at the origin with a radius $r$ large enough to contain $\partial \Omega$ and the support of $u$, put $\Omega_{\varepsilon}:=\left(\Omega \cap B_{r}(0)\right) \backslash B_{\varepsilon}(y)$, we have $R(\cdot, y) \in L_{2}\left(\rho_{2} ; \Omega_{\varepsilon}\right)$ and $P(\cdot, y) \in \mathscr{H}^{1,0}\left(\Omega_{\varepsilon}\right)$. Applying the second Green identity (8) in $\Omega_{\varepsilon}$ with $v=P(y, \cdot)$ and taking usual limits as $\varepsilon \rightarrow 0$, cf. [11], we get the third Green identity in $\Omega_{r}:=\Omega \cap B_{r}(0)$,

$$
u+\mathscr{R} u-V\left(T^{+} u\right)+W\left(\gamma^{+} u\right)=\mathscr{P} A u
$$

for $u \in \mathscr{D}(\bar{\Omega})$. Here,

$$
\mathscr{P} g(y):=\int_{\Omega} P(x, y) g(x) d x, \quad \mathscr{R} g(y):=\int_{\Omega} R(x, y) g(x) d x, \quad y \in \mathbb{R}^{2},
$$

are, respectively, the parametrix-based Newtonian and remainder potentials, while

$V g(y):=-\int_{\partial \Omega} P(x, y) g(x) d S_{x}, \quad W g(y):=-\int_{\partial \Omega}\left[T_{x} P(x, y)\right] g(x) d S_{x}, x \in \mathbb{R}^{2} \backslash \partial \Omega$,

are the parametrix-based single layer and double layer potentials. Deducing (13) we took into account that $u \equiv 0$ in $\Omega \backslash B_{r}(0) \subset \Omega \backslash \operatorname{supp} u$. Since no term in (13) depends on $r$ if $r$ is sufficiently large, we obtain that (13) is valid in the whole domain $\Omega$ for any $u \in \mathscr{D}(\bar{\Omega})$.

From definitions (11)-(12) and (14)-(15) one can obtain representations of the parametrix-based potential operators in terms of their counterparts for $a=1$ (i.e., associated with the Laplace operator $\Delta$ ), cf. [3, 4],

$$
\mathscr{P} g=\frac{1}{a} \mathscr{P}_{\Delta} g, \mathscr{R} g=-\frac{1}{a} \sum_{j=1}^{2} \partial_{j}\left[\mathscr{P}_{\Delta}\left(g \partial_{j} a\right)\right], V g=\frac{1}{a} V_{\Delta} g, W g=\frac{1}{a} W_{\Delta}(a g)
$$

The Newtonian and the remainder potential operators given by (14) for $\Omega=\mathbb{R}^{2}$ will be denoted as $\mathbf{P}$ and $\mathbf{R}$, respectively, and the relations similar to (16) hold for them as well.

In addition to conditions (4) and (5) on the coefficient $a$, we will sometimes also need the condition

$$
\rho_{2}^{2} \Delta a \in L_{\infty}\left(\mathbb{R}^{2}\right)
$$


Employing that the corresponding mapping properties hold true for the potentials associated with the Laplace operator $\Delta$, cf. eg. Section 8 in [13] and references therein, relations (16) lead to the following assertion.

Theorem 3. The following operators are continuous under conditions (5).

$$
\begin{aligned}
\boldsymbol{P} & : \mathscr{H}^{-1}\left(\mathbb{R}^{2}\right) \perp \mathbb{R} \rightarrow \mathscr{H}^{1}\left(\mathbb{R}^{2}\right), \\
\mathscr{P} & : \mathscr{H}^{-1}(\Omega) \perp \mathbb{R} \rightarrow \mathscr{H}^{1}\left(\mathbb{R}^{2}\right), \\
\boldsymbol{R} & : L_{2}\left(w ; \mathbb{R}^{2}\right) \rightarrow \mathscr{H}^{1}\left(\mathbb{R}^{2}\right), \\
V & : H_{*}^{-\frac{1}{2}}(\partial \Omega) \rightarrow \mathscr{H}^{1}(\Omega), \\
W & : H^{\frac{1}{2}}(\partial \Omega) \rightarrow \mathscr{H}^{1}(\Omega),
\end{aligned}
$$

while the following operators are continuous under conditions (5) and (17).

$$
\begin{aligned}
\mathscr{P} & : L_{2}\left(\rho_{2} ; \Omega\right) \perp \mathbb{R} \rightarrow \mathscr{H}^{1,0}\left(\mathbb{R}^{2} ; A\right), \\
\mathscr{R} & : \mathscr{H}^{1}(\Omega) \rightarrow \mathscr{H}^{1,0}(\Omega ; A), \\
V & : H_{*}^{-\frac{1}{2}}(\partial \Omega) \rightarrow \mathscr{H}^{1,0}(\Omega ; A), \\
W & : H^{\frac{1}{2}}(\partial \Omega) \rightarrow \mathscr{H}^{1,0}(\Omega ; A) .
\end{aligned}
$$

Similar to [10, Theorem 3.12] one can prove that $\mathscr{D}(\bar{\Omega})$ is dense in $\mathscr{H}^{1,0}(\Omega ; A)$ and in $\mathscr{H}^{1,0 \perp}(\Omega ; A)$. Then Theorem 3 and Lemma 2 imply the following assertion.

Corollary 1. The third Green identity (13) holds true for any $u \in \mathscr{H}^{1,0 \perp}(\Omega ; A)$.

The boundary integral (pseudo-differential) operators of the direct values and of the co-normal derivatives of the single and double layer potentials are defined by

$$
\begin{array}{cl}
\mathscr{V} g(y):=-\int_{\Gamma} P(x, y) g(x) d s_{x}, \quad \mathscr{W} g(y):=-\int_{\Gamma} T_{x} P(x, y) g(x) d s_{x} \quad y \in \Gamma, \\
\mathscr{W}^{\prime} g(y):=-\int_{\Gamma} T_{y} P(x, y) g(x) d s_{x} \quad \mathscr{L}^{ \pm} g(y):=T_{y}^{ \pm} W g(y) \quad y \in \Gamma .
\end{array}
$$

Applying the trace and co-normal derivative operators to the third Green identity (13), and using the jump relations for the potential operators we obtain for $u \in$ $\mathscr{H}^{1,0 \perp}(\Omega ; A)$,

$$
\begin{gathered}
\frac{1}{2} \gamma^{+} u+\gamma^{+} \mathscr{R} u-\mathscr{V} T^{+} u+\mathscr{W} \gamma^{+} u=\gamma^{+} \mathscr{P} A u \quad \text { on } \partial \Omega, \\
\frac{1}{2} T^{+} u+T^{+} \mathscr{R} u-\mathscr{W}^{\prime} T^{+} u+\mathscr{L}^{+} \gamma^{+} u=T^{+} \mathscr{P} A u \quad \text { on } \partial \Omega .
\end{gathered}
$$

Conditions (5) are assumed to hold for (27) and conditions (5) and (17) for (28).

For some functions $f, \Psi$ and $\Phi$ let us consider a more general indirect integral relation associated with equation (13). 


$$
u+\mathscr{R} u-V \Psi+W \Phi=\mathscr{P} f \quad \text { in } \Omega .
$$

Lemma 3. Let $u \in \mathscr{H}^{1,0 \perp}(\Omega ; A), f \in L_{2}\left(\rho_{2} ; \Omega\right) \perp \mathbb{R}, \Psi \in H_{*}^{-\frac{1}{2}}(\partial \Omega)$, and $\Phi \in$ $H^{\frac{1}{2}}(\partial \Omega)$ satisfy equation (29) and let conditions (5), (17) hold. Then, $u$ is a solution of the equation

$$
A u=f \quad \text { in } \Omega \text {, }
$$

while

$$
V\left(\Psi-T^{+} u\right)-W\left(\Phi-\gamma^{+} u\right)=0, \quad \text { in } \Omega .
$$

Proof. Since $u \in \mathscr{H}^{1,0 \perp}(\Omega ; A)$, we can write the third Green identity (13) for the function $u$. Then subtracting (29) from it, we obtain

$$
-V \Psi^{*}+W \Phi^{*}=\mathscr{P}[A u-f] \quad \text { in } \Omega,
$$

where $\Psi^{*}:=T^{+} u-\Psi$ and $\Phi^{*}:=\gamma^{+} u-\Phi$. Multiplying equality (32) by $a(y)$ we get

$$
-V_{\Delta} \Psi^{*}+W_{\Delta}\left(a \Phi^{*}\right)=\mathscr{P}_{\Delta}[A u-f] \quad \text { in } \Omega .
$$

Applying the Laplace operator $\Delta$ to the last equation and taking into consideration that both functions in the left-hand side are harmonic potentials, while the right-hand side function is the classical Newtonian potential, we arrive at Eq. (30). Substituting (30) back into (32) leads to (31).

Lemma 4. Let conditions (5) and (17) hold.

(i) If $\Psi^{*} \in H_{*}^{-\frac{1}{2}}(\partial \Omega)$ and $V \Psi^{*}=0$ in $\Omega$, then $\Psi^{*}=0$.

(ii) If $\Phi^{*} \in H^{\frac{1}{2}}(\partial \Omega)$ and $W \Phi^{*}(y)=0$ in $\Omega$, then $\Phi^{*}(x)=C / a(x)$, where $C$ is a constant.

Proof. The proof of item (i) coincides with the proof of its counterpart for interior domains in ([6]), while the proof of item (ii) is similar to the proof for the 3D case in [4, Lemma 4.2].

\section{BDIEs for Exterior Dirichlet BVP}

To reduce the variable-coefficient Dirichlet BVP (9)-(10) to a segregated boundarydomain integral equation systems, let us denote the unknown conormal derivative as $\psi:=T^{+} u \in H^{-\frac{1}{2}}(\partial \Omega)$ and further consider $\psi$ as formally independent of $u$.

For a given function $f$ in $L_{2}\left(\rho_{2} ; \Omega\right) \perp \mathbb{R}$, assume that the function $u$ satisfies the PDE $A u=f$ in $\Omega$. Then by substituting the Dirichlet condition into the third Green identity (13) and either into its trace (27) or into its co-normal derivative (28) on $\partial \Omega$, we can reduce the BVP (9)-(10) to two different systems of BoundaryDomain Integral Equations for the unknown functions $u \in \mathscr{H}^{1}(\Omega ; A)$ and $\psi:=$ $T^{+} u \in H^{-\frac{1}{2}}(\partial \Omega)$. 
BDIE system (D1) obtained under conditions (5) from the third Green's identity (13) and its trace equation (27) is

$$
\begin{aligned}
u+\mathscr{R} u-V \psi & =F_{0} \quad \text { in } \Omega, \\
\gamma^{+} \mathscr{R} u-\mathscr{V} \psi & =\gamma^{+} F_{0}-\varphi_{0} \quad \text { on } \partial \Omega,
\end{aligned}
$$

where

$$
F_{0}:=\mathscr{P} f-W \varphi_{0} \quad \text { in } \Omega .
$$

The system can be written in a matrix form as $\mathfrak{D}^{1} \mathscr{U}=\mathscr{F}^{1}$, where

$$
\mathscr{U}:=[u, \psi]^{t} \in \mathscr{H}^{1,0}(\Omega ; A) \times H^{-\frac{1}{2}}(\partial \Omega),
$$

and

$$
\mathfrak{D}^{1}:=\left[\begin{array}{cc}
I+\mathscr{R} & -V \\
\gamma^{+} \mathscr{R} & -\mathscr{V}
\end{array}\right], \mathscr{F}^{1}=\left[\begin{array}{c}
F_{0} \\
\gamma^{+} F_{0}-\varphi_{0}
\end{array}\right]
$$

From the mapping properties of $W$ and $\mathscr{P}$ in Theorem 3, we get the inclusion $F_{0} \in \mathscr{H}^{1,0}(\Omega ; A)$, and the trace theorem implies $\gamma^{+} F_{0} \in H^{\frac{1}{2}}(\partial \Omega)$. Therefore, $\mathscr{F}^{1} \in \mathscr{H}^{1}(\Omega) \times H^{\frac{1}{2}}(\partial \Omega)$.

BDIE system (D2) obtained under conditions (5) and (17) form the third Green's identity (13) and its co-normal derivative equation (28) is

$$
\begin{aligned}
u+\mathscr{R} u-V \psi & =F_{0} \quad \text { in } \Omega, \\
\frac{1}{2} \psi+T^{+} \mathscr{R} u-\mathscr{W}^{\prime} \psi & =T^{+} F_{0} \quad \text { on } \partial \Omega,
\end{aligned}
$$

where $F_{0}$ is given by (33). In a matrix form it can be written as $\mathfrak{D}^{2} \mathscr{U}=\mathscr{F}^{2}$, where

$$
\mathfrak{D}^{2}=\left[\begin{array}{cc}
I+\mathscr{R} & -V \\
T^{+} \mathscr{R} & \frac{1}{2} I-\mathscr{W}^{\prime}
\end{array}\right], \quad \mathscr{F}^{2}=\left[\begin{array}{c}
F_{0} \\
T^{+} F_{0}
\end{array}\right] .
$$

Note that the operator $\mathfrak{D}^{2}: \mathscr{H}^{1,0}(\Omega ; A) \times H_{*}^{-\frac{1}{2}}(\partial \Omega) \rightarrow \mathscr{H}^{1,0}(\Omega ; A) \times H^{-\frac{1}{2}}(\partial \Omega)$ is bounded.

\section{Equivalence and Uniqueness Theorems}

Theorem 4. Let $\varphi_{0} \in H^{\frac{1}{2}}(\partial \Omega), f \in L_{2}\left(\rho_{2} ; \Omega\right) \perp \mathbb{R}$, and conditions (5) and (17) hold.

(i) If some $u \in \mathscr{H}^{1,0 \perp}(\Omega ; A)$ solves the $B V P(9)-(10)$, then the pair $(u, \psi)$, where

$$
\psi=T^{+} u \in H_{*}^{-\frac{1}{2}}(\partial \Omega)
$$


solves BDIE systems (D1) and (D2).

(ii) If a pair $(u, \psi) \in \mathscr{H}^{1,0 \perp}(\Omega ; A) \times H_{*}^{-\frac{1}{2}}(\partial \Omega)$ solves BDIE system (D1), then $u$ solves BDIE system (D2) and BVP (9)-(10), this solution is unique, and $\psi$ satisfies (35).

Proof. (i) Setting $\psi:=T^{+} u$ and recalling how BDIE system (D1) and (D2) were constructed, we obtain that the couple $(u, \psi)$ solves them.

(ii) Let now a pair $(u, \psi) \in \mathscr{H}^{1,0 \perp}(\Omega ; A) \times H_{*}^{-\frac{1}{2}}(\partial \Omega)$ solves system (D1). Due to the first equation in the BDIE systems, the hypotheses of Lemma 3 are satisfied implying that $u$ solves $\operatorname{PDE}(9)$ in $\Omega$ and

$$
V\left(\psi-T^{+} u\right)-W\left(\varphi_{0}-\gamma^{+} u\right)=0 \quad \text { in } \Omega .
$$

Taking the trace of the first equation in (D1) and subtracting the second equation from it, we get $\gamma^{+} u=\varphi_{0}$ on $\partial \Omega$. Thus, the Dirichlet boundary condition is satisfied, and using this in (36), we obtain $V\left(\psi-T^{+} u\right)=0$ in $\Omega$. Lemma 4 (i) then implies $\psi=T^{+} u$.

The uniqueness of the BDIE system follows from the fact that the corresponding homogeneous BDIE systems can be associated with the homogeneous Dirichlet problem, which has only the trivial solution. Then the previous paragraph implies that the homogeneous BDIE system also have only the trivial solutions.

Theorem 5. Let $\varphi_{0} \in H^{\frac{1}{2}}(\partial \Omega), f \in L_{2}\left(\rho_{2} ; \Omega\right) \perp \mathbb{R}$, and conditions (5) and (17) hold.

(i) Homogeneous BDIE system (D2) admits only one linearly independent solution $\left(u^{0}, \psi^{0}\right) \in \mathscr{H}^{1,0 \perp}(\Omega ; A) \times H_{*}^{-\frac{1}{2}}(\partial \Omega)$, where $u^{0}$ is the solution of the Dirichlet $B V P$

$$
\begin{aligned}
A u^{0} & =0 \quad \text { in } \Omega, \\
\gamma^{+} u^{0} & =\frac{1}{a(x)} \quad \text { on } \partial \Omega,
\end{aligned}
$$

while

$$
\psi^{0}=T^{+} u^{0} \quad \text { on } \partial \Omega .
$$

(ii) The non-homogeneous BDIE system (D2) is solvable, and any of its solutions $(u, \psi) \in \mathscr{H}^{1,0 \perp}(\Omega ; A) \times H_{*}^{-\frac{1}{2}}(\partial \Omega)$ can be represented as

$$
u=\tilde{u}+C u^{0} \quad \text { in } \Omega
$$

where $\tilde{u}$ solves BVP (9)-(10) and C is a constant, while

$$
\psi=T^{+} \tilde{u}+C \psi^{0} \quad \text { on } \partial \Omega .
$$

Proof. Problem (37)-(38) is uniquely solvable in $\mathscr{H}^{1,0 \perp}(\Omega ; A)$ by Theorem 2. Consequently, the third Green identity (13) is applicable to $u^{0}$, leading to 


$$
u^{0}+\mathscr{R} u^{0}-V \psi^{0}=0 \quad \text { in } \Omega
$$

Taking the co-normal derivative of (42) and substituting (39) again, we arrive at

$$
\frac{1}{2} \psi^{0}+T^{+} \mathscr{R} u^{0}-\mathscr{W}^{\prime} \psi^{0}=0 \quad \text { on } \partial \Omega
$$

Equation (42) and (43) means that the pair $\left(u^{0}, \psi^{0}\right)$ solves the homogeneous BDIE system (D2).

To prove item (ii), we first remark that the solvability of non-homogeneous system (D2) follows from the solvability of the BVP (9)-(10) in $\mathscr{H}^{1,0 \perp}(\Omega ; A)$ and the deduction of system (D2).

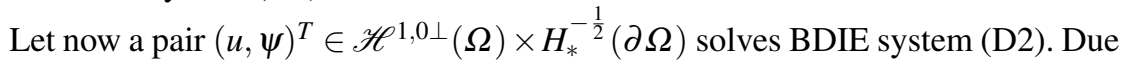
to the first equation in the BDIE systems, Lemma 3 implies that $u$ solves PDE (9) in $\Omega$ and relation (36) holds. Taking the co-normal derivative of the first equation in (D2) on $\partial \Omega$ and subtract it from the second equation in (D2), we obtain $\psi=T^{+} u$ on $\partial \Omega$. Then inserting this in (36) gives $W\left(\varphi_{0}-\gamma^{+} u\right)=0$, in $\Omega$, and Lemma 4(ii) implies

$$
\gamma^{+} u=\varphi_{0}+C / a(x) \quad \text { on } \partial \Omega
$$

where $C$ is a constant. Thus, $u$ satisfies the Dirichlet condition (44) instead of (10). Introducing the notation $\tilde{u}$ by (40) in (44) and taking into account (37)-(38) prove the claim of item (ii).

\section{References}

1. Amrouche, C. , Girault, V. and Giroire, J.: Weighted Sobolev Spaces for Laplace's Equation in $\mathbb{R}^{n}$. J. Math. Pures Appl.,73, 579-606 (1994).

2. Amrouche, C., Girault, V., and Schechter, : Dirichlet and Neumann exterior problems for the n-dimensional Laplace operator. An approach in weighted sobolev spaces. Journal de Mathématiques Pures et Appliquées,76, 55-81 (1997).

3. Chkadua, O., Mikhailov, S.E. and Natroshvili, D.: Analysis of direct boundary-domain integral equations for a mixed BVP with variable coefficient. I. Equivalence and invertibility. $J$. Integral Equations Appl., 21: 499-543 (2009).

4. Chkadua, O., Mikhailov, S.E. and Natroshvili, D.: Analysis of direct segregated boundarydomain integral equations for variable-coefficient mixed BVPs in exterior domains. Analysis and Applications, 11, No.4, No 4, 1350006 (2013).

5. Costabel, M.: Boundary integral operators on Lipschiz domains: Elementary results. SIAM J. Math. Anal., 19, 613-626 (1988).

6. Dufera, T.T. and Mikhailov, S.E.: Analysis of Boundary-Domain Integral Equations for Variable-Coefficient Drichlet BVP in 2D. In: Integral Methods in Science and Engineering: Computational and Analytic Aspects, Springer (Birkhäuser), Boston, 163-175, (2015).

7. Kufner, A. and Opic, B.: How to define reasonably weighted Sobolev spaces. Commentationes Mathematicae Universitatis Carolinae, 25, 537-554 (1984).

8. McLean, W.: Strongly Elliptic Systems and Boundary Integral Equations. Cambrige University Press, Cambrige, 2000.

9. Mikhailov, S.E.: Localized boundary-domain integral formulations for problems with variable coefficients. Eng. Anal. with Boundary El., 26, 681-690 (2002). 
10. Mikhailov, S.E.: Traces, extensions and co-normal derivatives for elliptic systems on Lipschiz domains. J. Math. Anal. Appl., 378, 324-342 (2011).

11. Miranda, C.: Partial differential equations of elliptic type. Springer, 1970.

12. Nédélec, J.C.: Acoustic and electromagnetic equations: integral representations for harmonic problems. Springer, 2001.

13. Sayas, F.-J., Selgas, V.: Variational views of stokeslets and stresslets. SeMA 63, 65-90 (2014). 